\title{
Impending Blindness by Obstructive Hydrocephalus Intractable to Endoscopic Third Ventriculostomy: Case Report
}

\author{
Nobuo Shirasaka ${ }^{1}$ (D) Muneyoshi Yasuda ${ }^{1} \cdot$ Kazuhito Takeuchi $^{2} \cdot$ Keisuke Ito $^{1} \cdot$ Mikiko Funai $^{1} \cdot$ Yohei Maruga $^{1} \cdot$ \\ Takeaki Totsuka $^{1} \cdot$ Yoshihito Hasegawa ${ }^{1} \cdot$ Akihiro Miyasaki $^{1} \cdot$ Makoto Negoro $^{1}$
}

Accepted: 2 December 2020 / Published online: 27 January 2021

(C) The Author(s) 2021

\begin{abstract}
Endoscopic third ventriculostomy (ETV) has been accepted for obstructive hydrocephalus (OH). We herein present a case of $\mathrm{OH}$ and optic nerve sheath edema (ONSE). Although ETV was successful, ONSE worsened. Discussion is focused on the relationship among intracranial hypertension (IH), ETV, and ONSE. The patient was a 19-year-old woman with complaints of headache and emesis. Papilledema was prominent. Radiological examination showed $\mathrm{OH}$ as an aqueductal tumor. Endoscopic tumorectomy and ETV were accomplished. Although headache and emesis disappeared, papilledema persisted. On the 4th day, the patient lost light perception. On magnetic resonance imaging, the third ventricular stroma and aqueduct were patent. Nevertheless, ONSE was prominent. After lumbar drainage, her vision was restored. Finally, ventriculoperitoneal shunting was performed, following which ONSE disappeared. IH is not always accompanied by ONSE. However, once it occurs, it may act separately from IH, and ETV may even exacerbate ONSE due to alteration of cerebrospinal fluid (CSF) flow into the chiasmatic cistern. In the optic nerve sheath, CSF pressure is not easily transmitted across different areas; this should explain the dissociation between ONSE and other symptoms of IH. Furthermore, we suggest that ONSE and acute optic dysfunction should be considered an independent crisis as "hydronervus opticus."
\end{abstract}

Keywords Blindness · Obstructive hydrocephalus $\cdot$ Endoscopic third ventriculostomy $\cdot$ Optic nerve sheath edema $\cdot$ Case report

\section{Introduction}

In the last decade, endoscopic third ventriculostomy (ETV) has been widely accepted as an alternative procedure to conventional ventriculoperitoneal shunting (VPS) for the treatment of patients with obstructive hydrocephalus $(\mathrm{OH})$ [1-5]. Patients who undergo ETV do not need device placement and can avoid slit ventricle syndrome, which is often seen after VPS. ETV is one of the ideal procedures available for treating $\mathrm{OH}$ caused by aqueductal tumor [6]. Although not as common, some patients with intracranial hypertension (IH)

This article is part of the Topical Collection on Surgery

Nobuo Shirasaka

shirasakanobuo@gmail.com

1 Department of Neurological Surgery, Ichinomiya Nishi Hospital, Taira, Kaimei, Ichinomiya, Aichi 494-0001, Japan

2 Department of Neurological Surgery, Nagoya University Hospital, Nagoya, Aichi, Japan demonstrate optic nerve sheath edema (ONSE), which is usually accompanied by papilledema and visual dysfunction [7-10].

In this paper, a case of $\mathrm{OH}$ is described. After the onset of headache and emesis, acute visual impairment gradually started. ETV was successful, but ONSE and optic dysfunction did not seize. Moreover, the patient suddenly became blind on the 4th day after ETV. Finally, additional cerebrospinal fluid (CSF) flow alteration was necessary. Importantly, the patient's pathophysiology is discussed in light of change in CSF pressure by ETV in the subarachnoid space of the optic nerve sheath.

\section{Case Presentation}

A 19-year-old female visited our hospital complaining of headache, fatigue, and emesis for the previous 10 days. She had noticed a change in vision a few days before the consultation.

On physical examination, her consciousness was clear with good orientation. Motor and sensory functioning of all extremities was normal. Her cranial nerves were well- 
functioning, except the optic nerves. She was not able to maintain visual focus on a document. Her visual acuity was 0.9 in the right eye and 0.1 in the left one. On fundoscopic evaluation (Fig. 1a, b), papilledema was serious in both eyes. No gaze palsy was noticeable.

The patient was immediately admitted to our hospital. Upon radiological examinations, computed tomography (Fig. 1c) of the brain revealed enlargement of the lateral and third ventricles. There was a round nodular lesion in the location of the aqueduct of the midbrain measuring $15 \mathrm{~mm}$ in diameter, and the density was partially high relative to the surrounding cerebral tissue. No calcified component was seen on or inside the lesion. Upon magnetic resonance imaging (MRI) (Fig. 1d-f), a round, nodular lesion in the location of the aqueduct of the midbrain was noted that was also $15 \mathrm{~mm}$ in diameter. It occupied the rostral end of the aqueduct and was of a low intensity on T1-weighted imaging (WI) and high intensity on T2WI, with a clear and smooth border. T2*WI disclosed low-intensity contents inside the nodule, suggesting old microbleeds. In addition, slight perilesional cerebral edema was observed. The lesion was partially enhanced with contrast medium (Fig. 1f). The presumptive diagnosis was tectal tumor including pilocytic astrocytoma (PA) and ependymoma, which had caused $\mathrm{OH}$. On the 2 nd day in the hospital, the visual dysfunction had worsened in that she could poorly read letters on a document. Horizontal nystagmus upon lateral gaze and right abducens nerve palsy were also apparent. The patient seemed somehow somnolent. Therefore, endoscopic surgery was performed on the 4th day in the hospital.

During the operation, under general anesthesia, a right frontal burr hole was made to insert a transparent plastic port into the anterior horn of the right lateral ventricle. A flexible endoscope was introduced into the third ventricle through the foramen of Monro. The rostral orifice of the aqueduct was completely obstructed by the tumor. It was soft and grayish purple in color, originating from the tectum of the midbrain. The tumor was removed totally in a piecemeal manner. Vascular structures were not significantly abundant in the tumor, and meticulous hemostasis was done by electrocautery. Finally, the aqueduct became patent down to the fourth ventricle. Then, ETV was also performed to assure CSF flow. The Liliequist membrane was not developed and the prepontine

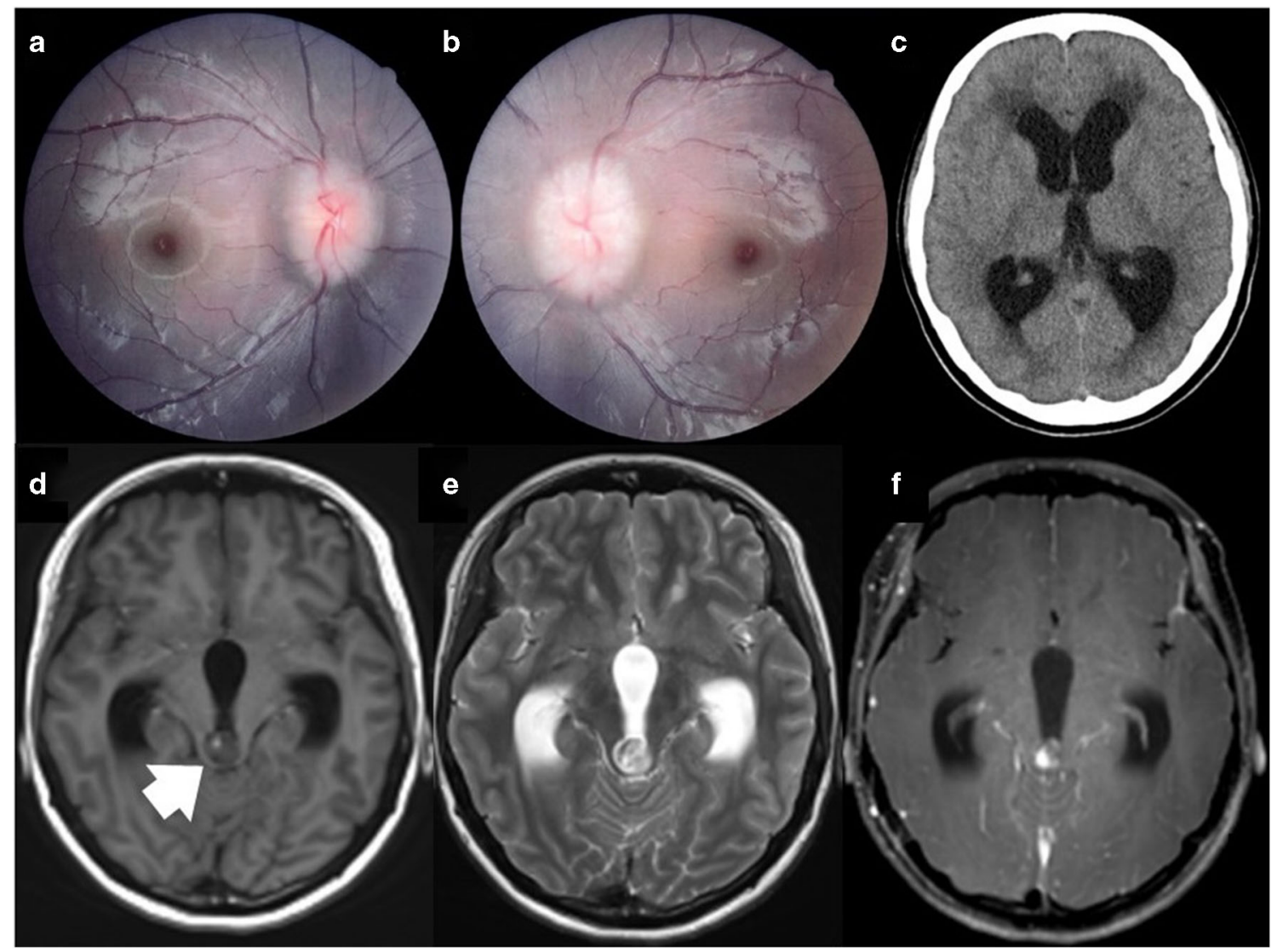

Fig. 1 Photographs of fundoscopic evaluation $(\mathbf{a}, \mathbf{b})$ were taken by an ophthalmologist on the first day of hospital admission. Papilledema is prominent in both eyes. Initial CT of the brain (c) shows enlargement of the lateral and third ventricles. On MRI before ETV, there is a round nodular lesion in the location of the aqueduct of the midbrain (d, arrow) that was of a low intensity on T1WI (d) and high intensity on T2WI (e), with a clear and smooth border. It measured $15 \mathrm{~mm}$ in diameter. Contrastenhanced T1WI disclosed partial enhancement inside the nodule (f) 
cistern was visible behind the posterior clinoid processes of the dorsum sellae. Because the ETV success score (ETVSS) was 90, no ventricular drainage was placed [11].

The endoscopic intervention was uneventful. The patient's headache and emesis disappeared immediately after the surgery; however, the visual disturbance was not alleviated at all during the next day. We held an optimistic perspective and expected that the patient's vision would improve within a few days. Therefore, the patient was observed conservatively in the hospital. However, her vision was not better throughout the following 2 days. Moreover, it suddenly worsened on the 4th day after the endoscopic surgery; the patient lost even her light perception. Bilateral papilledema was still prominent on fundoscopy. On MRI, CSF flow void was seen through the aqueduct and the third ventricle stoma, confirming their patency (Fig. 2), and the ventricular size seemed smaller. The floor of the third ventricle was not expanded anymore and was well-elevated (Fig. 2). All these findings suggested favorable relief of CSF pressure in the ventricles. Nevertheless, ONSE was still apparent in the orbital space (Fig. 3a). A steroid (methylprednisolone $1000 \mathrm{mg} /$ day for 3 days) was administered intravenously, and a continuous lumbar drainage apparatus was placed. The initial pressure was $40 \mathrm{cmH}_{2} 0$. Thereafter, her vision was restored slowly day by day. After 6 days of the lumbar drainage, she was able to read large letters. The lumbar drainage was kept in for 14 days, and more than $200 \mathrm{cc} /$ day of CSF needed to be drained to maintain pressure at $20 \mathrm{cmH}_{2} 0$. An analysis of CSF resulted in normal cell count and protein concentration findings without any evidence of infection or malignancy. Therefore, VPS was performed on the 15 th day after the endoscopic maneuver. At this point, her visual function improved significantly, enough for progressing through daily life. The patient was able to read newspapers at 7 days after the VPS (21 days after the initial surgery with ETV). The ONSE was observed to be significantly reduced on MRI at this point (Fig. 3b). She went home on foot on the 35 th day of hospitalization. The patient's visual acuity after 1 year was 0.7 in the right eye and 0.9 in the left one.

According to microscopic examination of the tumor tissue, the pathological diagnosis was PA (Fig. 4). No recurrence has been observed during regular radiological follow-up visits for 14 months.

\section{Discussion}

The importance of this case report is that ONSE still persisted with progressive blindness even after successful ETV, which ameliorated $\mathrm{IH}$ itself. Between ONSE and $\mathrm{IH}$, there was a significant disproportion of response to ETV.

ETV is regarded as the treatment of choice to relieve $\mathrm{OH}$. When $\mathrm{OH}$ is caused by an aqueductal tumor, both tumor removal and ETV are possible by endoscope $[13,14]$. The success rate of ETV is more than $70 \%$, which is high enough for clinical application [1-5]. In our current case, the ETVSS was 90 , representing a suitable figure supporting the expectation of clinical amelioration $[11,15]$. The ETV itself was successful. In addition, the aqueduct became patent at the end of the tumorectomy. Indeed, the headache with emesis disappeared immediately. Despite this desired clinical course, the patient suddenly became blind on the 4 th day. MRI revealed CSF flow void through the aqueduct and third ventricular stoma, suggesting their patency [16]. Intracranial findings on MRI suggested IH relief. However, bilateral papilledema and ONSE were still noticeable. In general, papilledema and ONSE are visible on MRI and are considered good indices of IH [17]. There are two hypothetical explanations for the dissociation: compound hydrocephalus and adaptation period. First, our case may have been compound hydrocephalus or a mixture of undiscovered CSF malabsorption and $\mathrm{OH}$. At least some $20 \%$ of $\mathrm{OH}$ patients do not respond to ETV [1-5], and
Fig. 2 Before ETV, sagittal T1WI MRI showed ballooning of the floor of the third ventricle (a, arrow). On the 4th day after ETV, sagittal T2WI MRI (b) disclosed an apparent CSF flow void through the aqueduct (white arrowhead) and stoma of the third ventricle (black arrowhead), confirming their patency. Note that the floor of the third ventricle is slack (arrow). All these findings indicate that ETV is successful

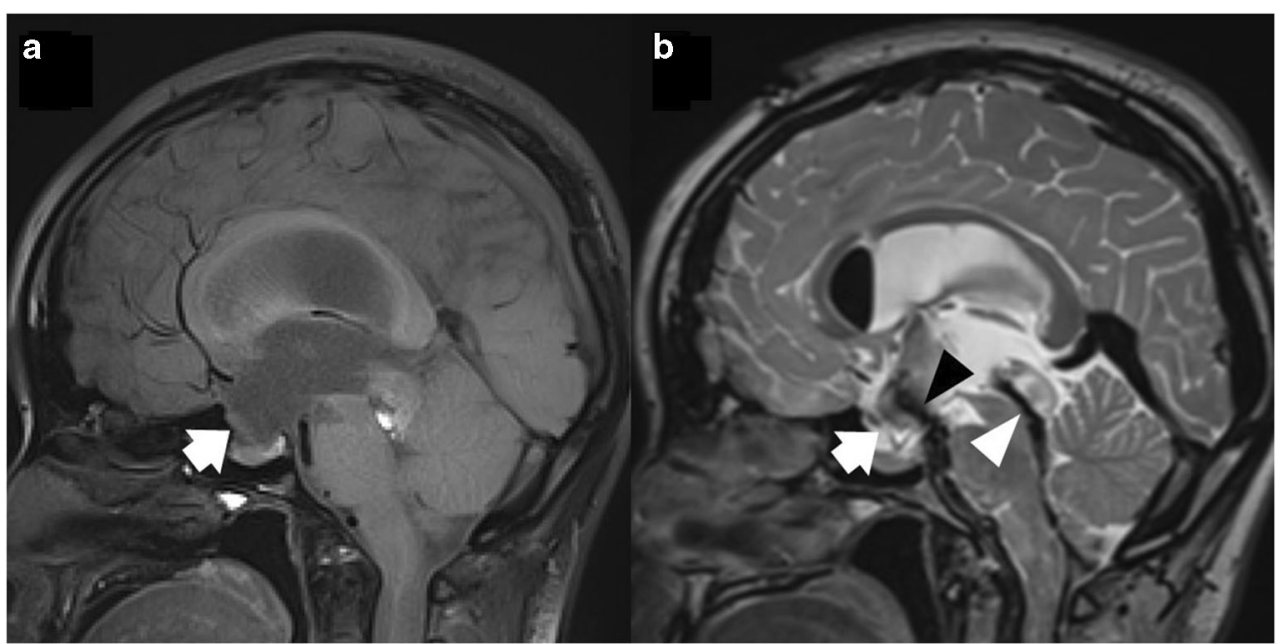




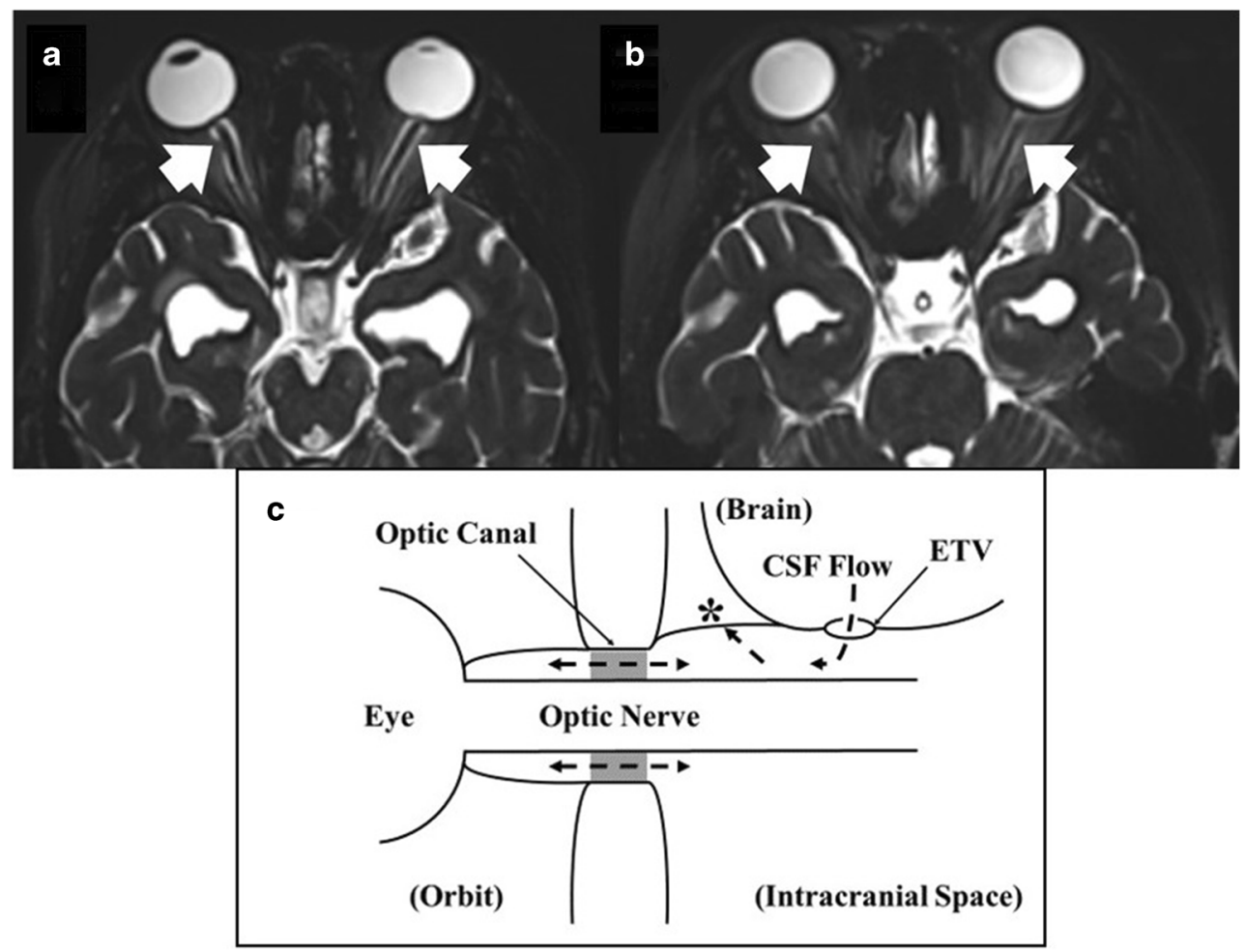

Fig. 3 On the 4th day after ETV, MRI constructive interference in a steady state (CISS, a) shows noticeable ONSE: CSF accumulation is observed around the optic nerve inside its sheath (a, arrow). MRI CISS obtained 9 days after VPS (27 days after ETV and 23 days after commencement of the lumbar drainage) disclosed a reduction of ONSE (b, arrow). Schematic explanation of CSF flow (dashed arrows) between the optic sheath and the intracranial space $(\mathbf{c})$. The arachnoid space extends from the cranium into the optic sheath through the optic canal. However, in the optic canal, abundant arachnoid trabeculae are present around the nerve and form a mesh zone. Therefore, intracranial CSF pressure is not easily transmitted to the optic sheath. Moreover, the chiasmatic cistern is generally sealed along its rostral side (asterisk) so that CSF may accumulate in the cistern after ETV $[7,12]$
Fig. 4 On light microscope, the tumor tissue is observed to be composed of two histological parts (biphasic pattern): compact and spongy. The compact part consisted of cells with bipolar processes (so-called hair-like cells) and has a relatively high cellular population. The spongy part is a hypocellular area with microcystic interstitial matrix. Vascular proliferation is observed in a glomeruloid fashion.

Although Rosenthal fibers or eosinophilic granular bodies are not so abundant in the tissue, the noted histological characteristics are compatible with those of pilocytic astrocytoma. GFAP(+), IDH1(R132H)(-), ATRX(+), p53(-), and Ki-67 2\%.

Hematoxylin and eosin stain, magnification $\times 100$

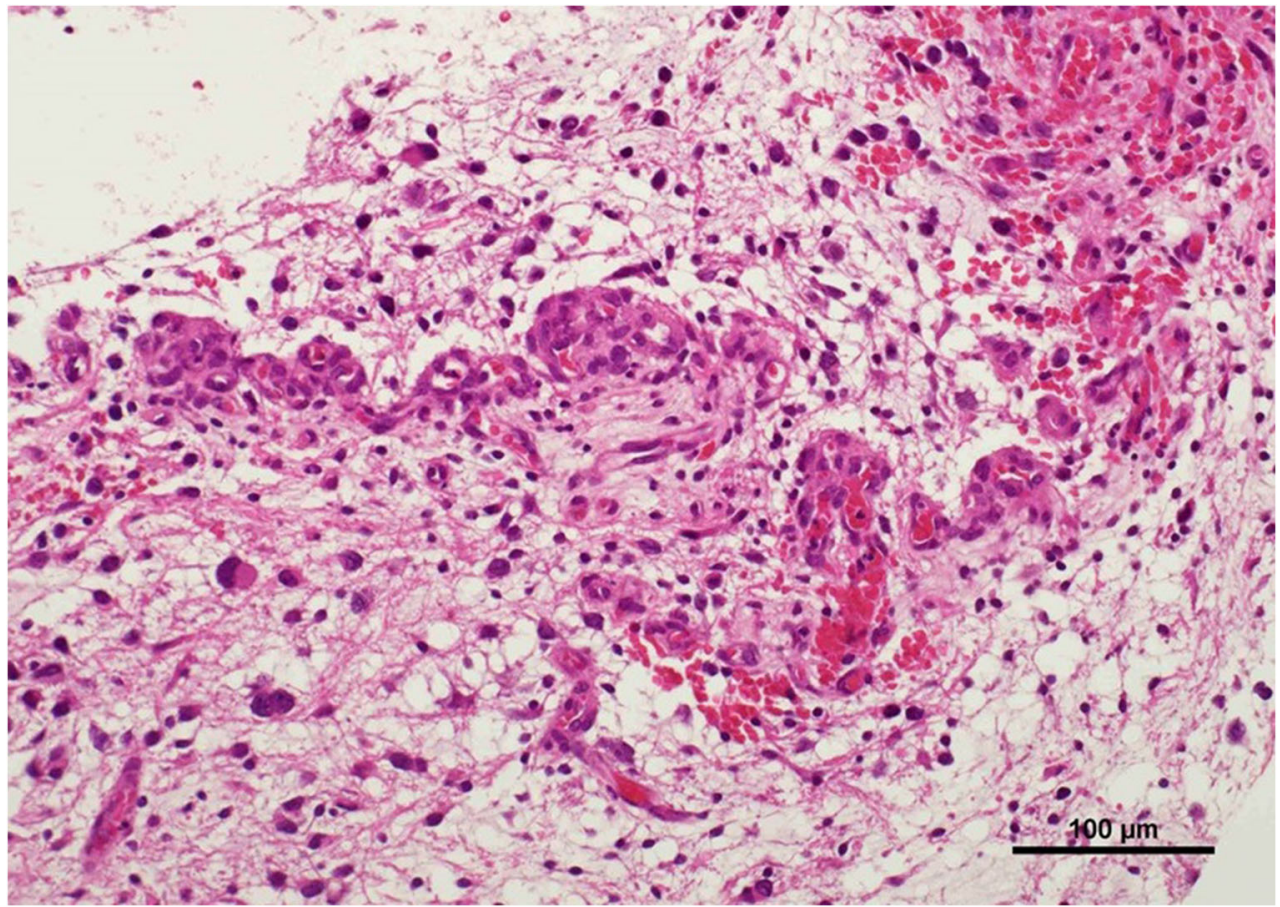


the reason for nonresponse is supposed to be compound hydrocephalus. In theory, ETV alone is not suitable for this entity. Second, this case may have required a long adaptation period. Immediately after ETV, the CSF flow diversion is often not adequate or steady, demanding a certain adaptation period that is variable in time $[18,19]$. In the present case, it may have been exceptionally long. Regardless of which explanation is valid, for this patient, it is clear that ETV alone could not sufficiently reduce the CSF pressure in the optic nerve sheath. This fact suggests that CSF pressure in the optic nerve sheath does not change simultaneously with and could remain even higher than that in the intracranial space.

Visual impairment with optic disk edema is well-known as an IH manifestation [20] but is not as common as usually assumed in the daily clinical practice of IH. In fact, only $12 \%$ of hydrocephalic patients, mainly by way of $\mathrm{OH}$, have visual impairment [8]. Optic function is not necessarily vulnerable to $\mathrm{IH}$, and intracranial symptoms such as headache, emesis, and somnolence usually precede visual loss. However, once IH starts to cause ONSE, which affects vision, it may persist or even deteriorate independently of the intracranial condition in certain cases.

ONSE is an accumulation of CSF in the arachnoid space of the optic nerve sheath inside the orbital space. The optic nerve there is surrounded by subarachnoid space extending from the intracranial arachnoid space through the optic canal. The CSF pressure of the optic sheath is regarded as identical to that of the intracranial subarachnoid space. However, in the optic canal, there are abundant arachnoid trabeculae around the nerve that form a mesh zone [21]. This structure demonstrates a wide individual variability or considerable laterality. Different from rodents and primates, humans often exhibit a highly dense mesh structure in this location [21] that often behaves like a "valve" or a "percolator" between the cranium and the optic sheath such that intracranial CSF pressure is not easily transmitted to the optic sheath. In addition, the chiasmatic cistern is tightly sealed by the arachnoid membrane along the superior surface of the optic nerve and the chiasm. Therefore, intracranial subarachnoid CSF flow may be blocked at this level even after ETV, resulting in high pressure at the orifice of the optic canal (Fig. 3c) [22]. These anatomical observations would explain why optic disk edema and visual loss are not so commonly observed with $\mathrm{IH}$, and there is a chronological and/or clinical discrepancy between the optic deterioration and other IH signs. Once ONSE and visual loss start with IH, they may persist or even worsen despite good relief of the original IH. In this situation, they should no longer be regarded as a part of $\mathrm{IH}$. We should be aware of the potential of ONSE with abruptly progressive optic dysfunction to be an independent clinical crisis termed as "hydronervus opticus."

When OH causes ONSE with acutely worsening blurred vision, ETV and/or aqueductal recanalization alone may not be helpful anymore to seize the ONSE. Our patient must have been in the acute phase of ONSE, for which aggressive CSF divergence should have been employed for optic rescue. ETV generally has a great therapeutic effect on $\mathrm{OH}$ without any doubt, but CSF flow rate and intracranial pressure are not intentionally controlled by ETV. Moreover, ETV may even worsen ONSE after a certain time, as described above. Therefore, for $\mathrm{OH}$ patients with acute visual impairment, external drainage should be maintained for at least several days after ETV. In addition, caring clinicians should be careful regarding acute visual loss for 1 week after ETV. Preservation or recovery of vision is one of the major goals in treating IH [17]. When visual deterioration is evident, an urgent countermeasure must be considered to reduce the optic nerve pressure for saving visual function. External CSF drainage and optic nerve sheath fenestration are worth considering [7, 12, 17, 23, 24].

Since this is a report of a single case, clinical evidence is limited to propose a final verdict on the relationship between ONCE and IH. It is necessary to analyze a large group of patients with similar conditions.

\section{Conclusion}

In this paper, we report a case of acute visual disturbance with ONSE by OH. Although ETV was effective for IH symptoms, ONSE persisted with visual deterioration, while lumbar drainage and VPS ameliorated it. Once IH starts to affect vision in ONSE, it may behave as a separate clinical entity from IH and we therefore should regard it as an independent clinical crisis as "hydronervus opticus" in certain cases. For $\mathrm{OH}$ patients having ONSE and abrupt blurred vision, ETV alone may not be enough and may even exacerbate them.

Acknowledgments The authors are grateful to Dr. Tsuyoshi Terashima and Dr. Dai Ikebe in the Department of Pathological Diagnosis at Ichinomiya Nishi Hospital for their help through pathological discussion. The authors would also like to thank Dr. Kozo Adachi and Dr. Tomohiro Mizuno in the Department of Ophthalmology at Ichinomiya Nishi Hospital for optic assessment of the case. Finally, we would like to express our deepest gratitude to Dr. Aritoshi Shirasaka at Hamamatsu Medical University for his sincere advice on clinical practice. The authors would like to thank Enago (www.enago.jp) for the English language review.

Authors' Contributions Nobuo Shirasaka: writing the manuscript, Muneyoshi Yasuda: analysis of the pathophysiology, Kazuhito Takeuchi: planning of the manuscript, Keisuke Ito: sampling of radiological data, Mikiko Funai: analysis of radiological data and illustration, Yohei Maruga: supervising clinical and surgical description, Takeaki Totsuka: data acquisition from medical records, Yoshihito Hasegawa: data acquisition from medical records, Akihiro Miyasaki: supervising discussion, Makoto Negoro: critic supervising.

Data Availability Not applicable. 


\section{Compliance with Ethical Standards}

Conflict of Interest The authors declare that they have no conflict of interest.

Ethics Approval Not applicable. This paper was constructed according to guidelines of the hospital for patients' privacy.

Consent to Participate The patient in this paper has signed a form of consent.

\section{Consent for Publication The patient has signed a form of consent.}

\section{Code Availability Not applicable.}

Open Access This article is licensed under a Creative Commons Attribution 4.0 International License, which permits use, sharing, adaptation, distribution and reproduction in any medium or format, as long as you give appropriate credit to the original author(s) and the source, provide a link to the Creative Commons licence, and indicate if changes were made. The images or other third party material in this article are included in the article's Creative Commons licence, unless indicated otherwise in a credit line to the material. If material is not included in the article's Creative Commons licence and your intended use is not permitted by statutory regulation or exceeds the permitted use, you will need to obtain permission directly from the copyright holder. To view a copy of this licence, visit http://creativecommons.org/licenses/by/4.0/.

\section{References}

1. Waqar M, Ellenbogen JR, Stovell MG, Al-Mahfoudh R, Mallucci C, Jenkinson MD. Long-term outcomes of endoscopic third ventriculostomy in adults. World Neurosurg. 2016;94:386-93.

2. Lam S, Harris DA, Lin Y, Rocque BG, Ham S, Pan IW. Outcomes of endoscopic third ventriculostomy in adults. J Clin Neurosci. 2016;31:166-71.

3. Isaacs AM, Bezchlibnyk YB, Yong H, Koshy D, Urbaneja G, Hader WJ, et al. Endoscopic third ventriculostomy for treatment of adult hydrocephalus: long-term follow-up of 163 patients. Neurosurg Focus. 2016;41:E3.

4. Grand W, Leonardo J, Chamczuk AJ, Korus AJ. Endoscopic third ventriculostomy in 250 adults with hydrocephalus: patient selection, outcomes, and complications. Neurosurgery. 2016;78:109-19.

5. Salvador SF, Oliveira J, Pereira J, Barros H, Vaz R. Endoscopic third ventriculostomy in the management of hydrocephalus: outcome analysis of 168 consecutive procedures. Clin Neurol Neurosurg. 2014;126:130-6.

6. Diaz RJ, Girgis FM, Hamiltonn MG. Endoscopic third ventriculostomy for hydrocephalus due to tectal glioma. Can J Neurol Sci. 2014;41:476-81.

7. Rigi M, Almarzouqi SJ, Morgan ML, Lee AG. Papilledema: epidemiology, etiology, and clinical management. Eye Brain. 2015;7: 47-57.

8. Jung JH, Chai YH, Jung S, Kim IY, Jang WY, Moon KS, et al. Visual outcome after endoscopic third ventriculostomy for hydrocephalus. Childs Nerv Syst. 2018;34:247-55.
9. Singhal A, Yang MM, Sargent MA, Cochrane DD. Does optic nerve sheath diameter on MRI decrease with clinically improved pediatric hydrocephalus? Childs Nerv Syst. 2013;29:269-74.

10. Rohr AC, Riedel C, Fruehauf MC, van Baalen A, Bartsch T, Hedderich J, et al. MR imaging findings in patients with secondary intracranial hypertension. AJNR Am J Neuroradiol. 2011;32:1021-9.

11. Kulkarni AV, Drake JM, Kestle JR, Mallucci CL, Sgouros S, Constantini S. Predicting who will benefit from endoscopic third ventriculostomy compared with shunt insertion in childhood hydrocephalus using the ETV success score. J Neurosurg Pediatr. 2010;6: $310-5$.

12. Fonseca PL, Rigamonti D, Miller NR, Subramanian PS. Visual outcomes of surgical intervention for pseudotumour cerebri: optic nerve sheath fenestration versus cerebrospinal fluid diversion. Br J Ophthalmol. 2014;98:1360-3.

13. Kobayashi N, Ogiwara H. Endoscopic third ventriculostomy for hydrocephalus in brainstem glioma: a case series. Childs Nerv Syst. 2016;32:1251-5.

14. O'Brien DF, Hayhurst C, Pizer B, Mallucci CL. Outcomes in patients undergoing single-trajectory endoscopic third ventriculostomy and endoscopic biopsy for midline tumors presenting with obstructive hydrocephalus. J Neurosurg. 2006;105:219-26.

15. Breimer GE, Sival DA, Brusse-Keizer MG, Hoving EW. An external validation of the ETVSS for both short-term and long-term predictive adequacy in 104 pediatric patients. Childs Nerv Syst. 2013;29:1305-11.

16. Udayakumaran S, Joseph T. Can we predict early endoscopic third ventriculostomy failure? The role of ultra-early postoperative magnetic resonance imaging in predicting early endoscopic third ventriculostomy failure. World Neurosurg X. 2019;2:100013.

17. Farb R, Rovira A. Hydrocephalus and CSF disorders. In: Hodler J, Kubik-Huch RA, von Schulthess GK, editors. Diseases of the brain, head and neck, spine 2020-2023: diagnostic imaging [internet]. Cham: Springer; 2020.

18. Santamarta D, Martin-Vallejo J. Evolution of intracranial pressure during the immediate postoperative period after endoscopic third ventriculostomy. Acta Neurochir Suppl. 2005;95:213-7.

19. Bellotti A, Rapana A, Iaccarino C, Schonauer M. Intracranial pressure monitoring after endoscopic third ventriculostomy: an effective method to manage the 'adaptation period'. Clin Neurol Neurosurg. 2001;103:223-7.

20. Shirasaka A, Furuya Y, Ryu H, Yokoyama T, Uemura K. Report of infantile intraventricular meningioma. No Shinkei Geka. 1987;15: 596-72.

21. Hayreh SS. Pathogenesis of optic disc edema in raised intracranial pressure. Prog Retin Eye Res. 2016;50:108-44.

22. Yassagil MG. Microneurosurgery, vol. 1. New York: ThiemeStratton; 1984. p. 31.

23. Jefferis JM, Raoof N, Carroll T, Salvi SM. Optic nerve sheath fenestration in patients with visual failure associated with vestibular schwannoma. Br J Neurosurg. 2019;33:402-8.

24. Agarwal MR, Yoo JH. Optic nerve sheath fenestration for vision preservation in idiopathic intracranial hypertension. Neurosurg Focus. 2007;23:E7.

Publisher's Note Springer Nature remains neutral with regard to jurisdictional claims in published maps and institutional affiliations. 